\title{
An Automatic Segmentation for Determination of IV Vessel Boundaries
}

\author{
Mazhar B. Tayel, M. A. Massoud, and Y. F. Shehata
}

\begin{abstract}
Coronary heart disease ranks as the top disease which causes the death in the Egypt. Intravascular (IV) ultrasound imaging is used along with $\mathrm{X}$-ray coronary angiography to detect vessel pathologies. This paper introduces three automated approaches for detection of lumen and media-adventitia borders in IV images. In the first method, a 2D median filter is used to initialize the lumen and media-adventitia contours. The second method uses the Lucy-Richardson method and the 2D median filter to determine the lumen border. Method three is a combination of two methods to combine their advantages. The catheter boundaries were automatically determined. This method shows a higher accuracy and ability to work under artifacts and noisy conditions.
\end{abstract}

Index Terms - Intravascular, segmentation, median filter, lucy-richardson method.

\section{INTRODUCTION}

According to the latest WHO data published in 2011 coronary heart deaths in Egypt reached or $21.73 \%$ of total dept. The age adjusted death rate was 173.98 per 100,000 of population ranks Egypt \#33 in the world and \#7 in the Middle East, not only but also coronary heart disease is rank \#1 of Top 20 Causes of Death in Egypt [1]. Because of the death rate in Egypt, research effort must be directed to decrease this rate by early determination of coronary heart disease.

Intravascular ultrasonic (IVUS) examinations are generally performed prior to vessel intervention treatments, such as balloon angioplasty, atherectomy, and rotablator, laser or stent deployment [2].

IVUS exam is a catheter-based technique that renders 2D cross-sectional image of the coronary arteries, and provides information concerning the lumen and wall morphology [3].

IVUS used along with X-ray coronary angiography to detect vessel pathologies [4]. In the IV, vessel is characterized by two distinct borders: the lumen border which corresponds the lumen -wall interface, and the mediaadventitia border which represents the boundary between the media and adventitia [5]. Fig. 1 shows the Lumen and Media-adventitia borders of typical IVUS image.

Segmentation of arterial wall boundaries is still an important problem. Much researches have been done to give better segmentation result for better diagnostics, evaluation

Manuscript received January 1, 2014; revised April 18, 2014.

Mazhar B. Tayel is with the Faculty of Engineering, Alexandria University, Egypt.

M. A. Massoud is with the Department of Biomedical Engineering, El-Minia University, Egypt (e-mail: dr.massoud@mu.edu.eg).

Y. F. Shehata is with the Department of Computer and System Engineering, El-Minia University, Egypt (e-mail: eng.yasseen.farouk@mu.edu.eg). and therapy planning.

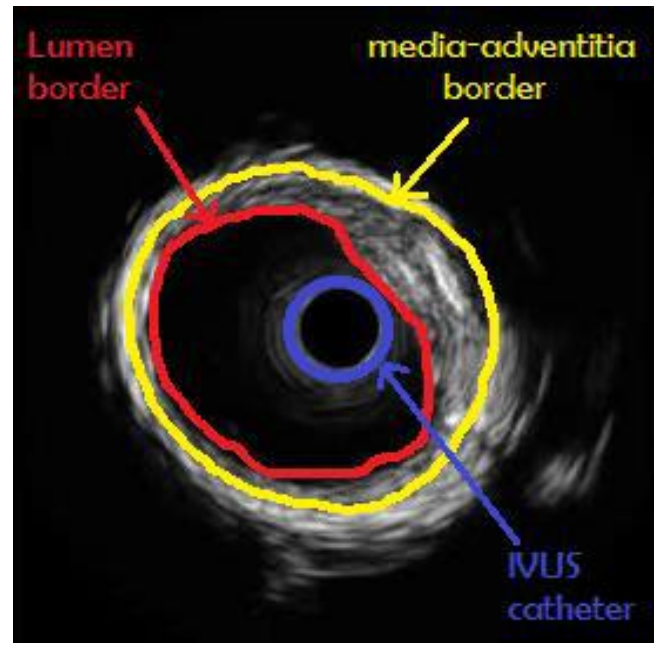

Fig. 1. A typical IVUS image.

The previous segmentation of IVUS works can be divided in many methods. These methods are based on automated methods, output of segmentation methods, and techniques used for segmentation.

Commonly the segmentation of an IVUS image is performed manually by an expert. When some kinds of user interaction are required, semi-automatic methods are used [6]-[14]. Automatic methods are used when the user interaction isn't required [15]-[20].

In the output of segment process, several techniques have been proposed for the automatic IVUS border segmentation. Automatic IVUS border segmentation methods can be designed to segment the borders such as: Lumen border [21], Media-adventitial border [22], [23], or both of the lumen and the Media-adventitial borders [3].

According to segment process techniques, there are many ways introduced. As edge detection techniques, in [18], Canny edge detection with different sigma is used to segment IVUS borders. Also a texture information extraction technique is used to segment IVUS images, in [3], the author uses multilevel discrete wavelet frames decomposition along with the intensity information for contour initialization. In the same year, [19] uses a discrete wavelet packet transform along with k-means clustering for contour initialization.

Probabilistic techniques also applied for segmentations, [13] uses the minimization of a probabilistic cost function using gray level histograms for lumen identification.

As a machine learning approach, in [11], a Hopfield neural network is used to modify and minimize the energy function; in [24], data sets are used to learn what pixels the human observer most often chooses for border pixels, then uses the learned system to detect border. 
The median filter is particularly good at removing outliers and is edge-preserving [25], in IVUS image analysis the median filter was usually used in preprocessing of the image [4], in [26], the median filter was used for filtering the initial contour.

Finally, analysis applied to IVUS images not only for detecting borders but also for detecting plaques, identifing types of plaques, and identifing bifurcations [27].

\section{METHODS}

Three different methods are introduced in this work.

\section{A. The First Introduced Method}

The first proposed algorithm for detection of lumen and media-adventitia borders in IVUS is shown in Fig. 2.

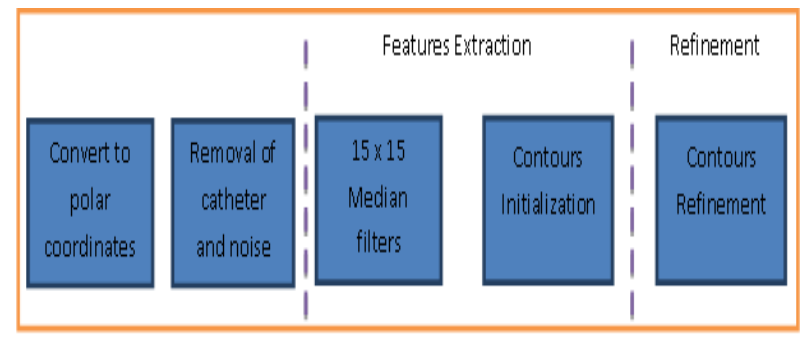

Fig. 2. The flow chart of the first method.

The IVUS image contours detection is obtained by applying two sub-stages. The first one is conversion of an image into polar coordinates and the second one is removal of the catheter and noise.

The IVUS images include not only tissue and blood regions, but also the outer boundary of the catheter itself. The latter defines a dead zone of radius equal to that of catheter, where no useful information is contained. The following equation is used to remove the catheter-induced artifacts;

$$
I(r, \theta)=0, \text { for } r<\left(\frac{D}{2}+d\right)
$$

where $I$ is intensity at coordinate point $(\mathrm{r}, \theta), \mathrm{D}$ is the diameter of catheter, and $\mathrm{d}$ is a small constant[3].

Results of preprocessing steps are shown in Fig. 3.
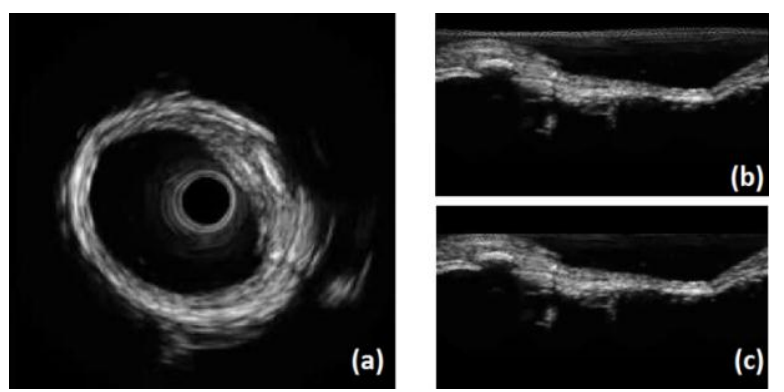

Fig. 2. Original IVUS image (a) and the corresponding polar coordinate images before (b) and after (c) the removal of catheter-induced artifacts.

\section{1) Features extraction}

\section{- Lumen contour initialization:}

The lumen border is a border between dark region and semi-dark region of an image. The following algorithm is used to determine the lumen border.

1) Find the value of $r$ corresponding to each $\theta$ degree at $I(\theta$, $r)$
2) If the value of gray level pixel of $r$ is higher than the minimum intensity value of the wall of vessel.

3) Then save it in the border array under index of $\theta$.

4) The initial contour of the lumen border is a 360 values $\{r 1$, $r 2, r 3, \ldots \ldots, r 360\}$ vector.

\section{- Media-adventitia contour initialization:}

The high intensity pixels in the first region was replaced by the average of pixels intensity, and then apply the 2D Median filter with $[15 \times 15]$ mask to smooth the image, then search about pixels that are most like to the media-adventitial border by applying the following algorithm:

1) Trace each $\theta$ degree in $I(\theta, r)$

2) Smoothing data of $I(\theta$, all $)$ and save it in temp vector.

3) Find the max value in the temp vector and its index

4) Then save this index value in the border array under index of $\theta$.

The initial contour of the media-adventitia border is a 360 values $\{r 1, r 2, r 3, \ldots \ldots, r 360\}$ vector.

\section{2) Refinement}

For Lumen contour fast refinement, a smooth operation by $5 \times 5$ windows is applied and repeated five times. For accurate refinement of the media-adventitia border, the following mean value formula is applied to initial point which has smaller value than a boundary of media-adventitia contour.

$$
T(i)=\frac{(\max (L)+d)+L(i)}{2}
$$

where $L$ is the set of points of the Lumen contour, $T$ is the limit contour, and the $d$ is a small constant value.

A $7 \times 7$ smooth window is applied and repeated 20 times to smooth the contour. Finally the average of limit value is taken to find the refinement Media-adventitia contour.

\section{B. The Second Introduced Method}

In this method the de-blurring filter using Lucy-Richardson with $7 \times 7$ mask and $2 \mathrm{D}$ filter with $15 \times 15$ mask are applied simultaneously before the preprocessing of method (1). After preprocessing, the algorithm completes steps as method (1). The flowchart of method (2) is illustrated in Fig. 4.

\section{The Third Introduced Method}

This method is a combination of the two previous methods, method (2) is better than method (1) in detecting the lumen border, and method (1) is better than method (2) in detecting the media-adventitial border, so method (3) improves detection of lumen and media-adventitial borders. The flowchart of this method is composed of two channels as shown in Fig. 5.

\section{1) Channel (1)}

1) Convert the image from Cartesian to polar.

2) Apply the $2 \mathrm{D}$ median filter with $15 \times 15$ mask.

3) Trace the lumen border using the IVUS saved image.

\section{2) Channel (2)}

1) Apply the de-blur image using the Lucy-Richardson method,

2) Apply the $2 \mathrm{D}$ median filter with $15 \times 15$ mask,

3) Convert the image from Cartesian to polar, and 
4) Trace the media-adventitial border using the IVUS saved image.

After completing the steps of the two channels, refinement contours of lumen border and media-adventitial border are begun, and followed by the conversion image from polar to Cartesian coordinate. The previous steps are repeated for each frame.

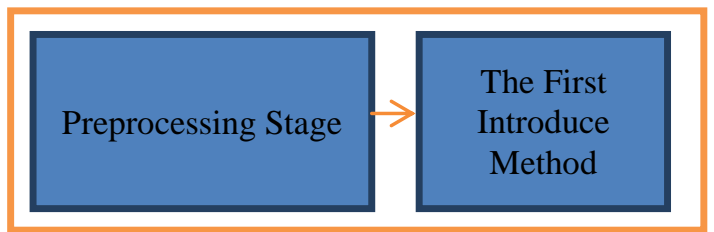

a) The block diagram of the second method.

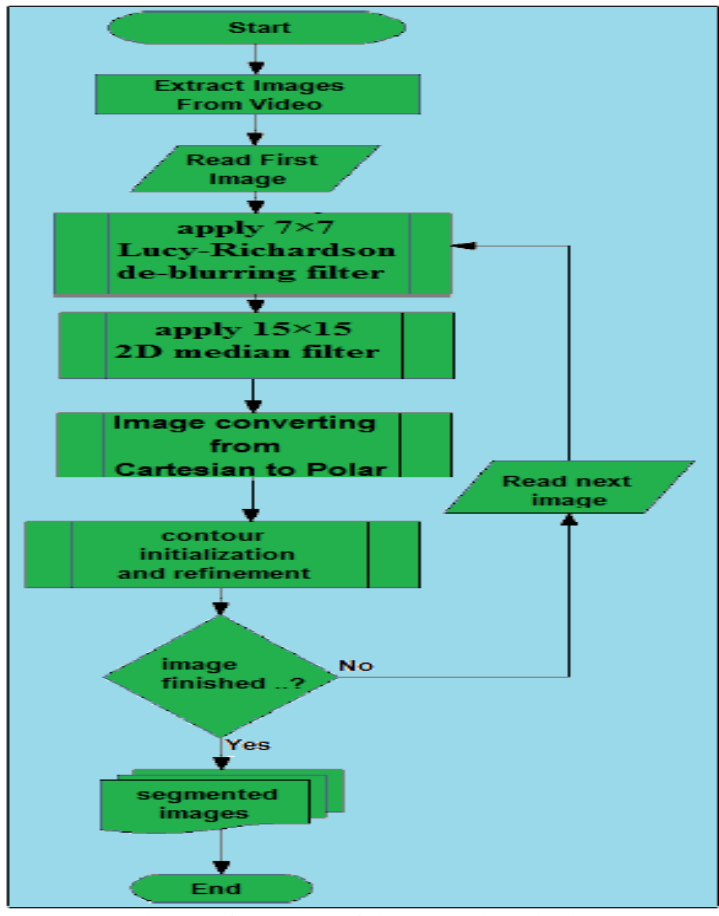

b) The flowchart of the second method.

Fig. 4. The block diagram and flowchart of the second method.

\section{Test of the Algorithm}

The Bland-Altman and the linear regression plots are common statistical analysis methods to evaluate vessel segmentation in IVUS imaging. Finally, performance factors like the mean, the minimum, and the maximum of the error are very important to compare the methods with each other.

The test of the algorithm was implemented on Samsung computer with OS Windows 7, a $2.53 \mathrm{GHz}$ Intel Core i3 processor, and a 4GB RAM. The program was written and executed in MATLAB, version 7.8.0.347 (R2009a) 64-bit software.

The test of the algorithm consists of three stages: manual table stage, automatic table stage, and statistical analysis stage.

\section{1) Manual table stage}

This stage is applied to calculate the data of lumen and vessel taken manually by expert doctor.

After scanning the image, the following steps have been done;

1) Read the image with red contour as the lumen border and green contour as media-adventitial border.
2) Trace the red pixels to detect the lumen border. Convert the red pixels to white pixels by threshold, and fill the area inside this contour. Then determine the area of the white object, and save it as the lumen cross section area (LCSA) according to this image.

3) Apply the previous step for green pixels which represent the media-adventitial border to determine the vessel cross section area (VCSA).

4) Calculate the vessel wall cross section area (WCSA) by Subtract LCSA from VCSA. Then store them as one line on table named manual table.

5) Repeat the previous steps for each frame.

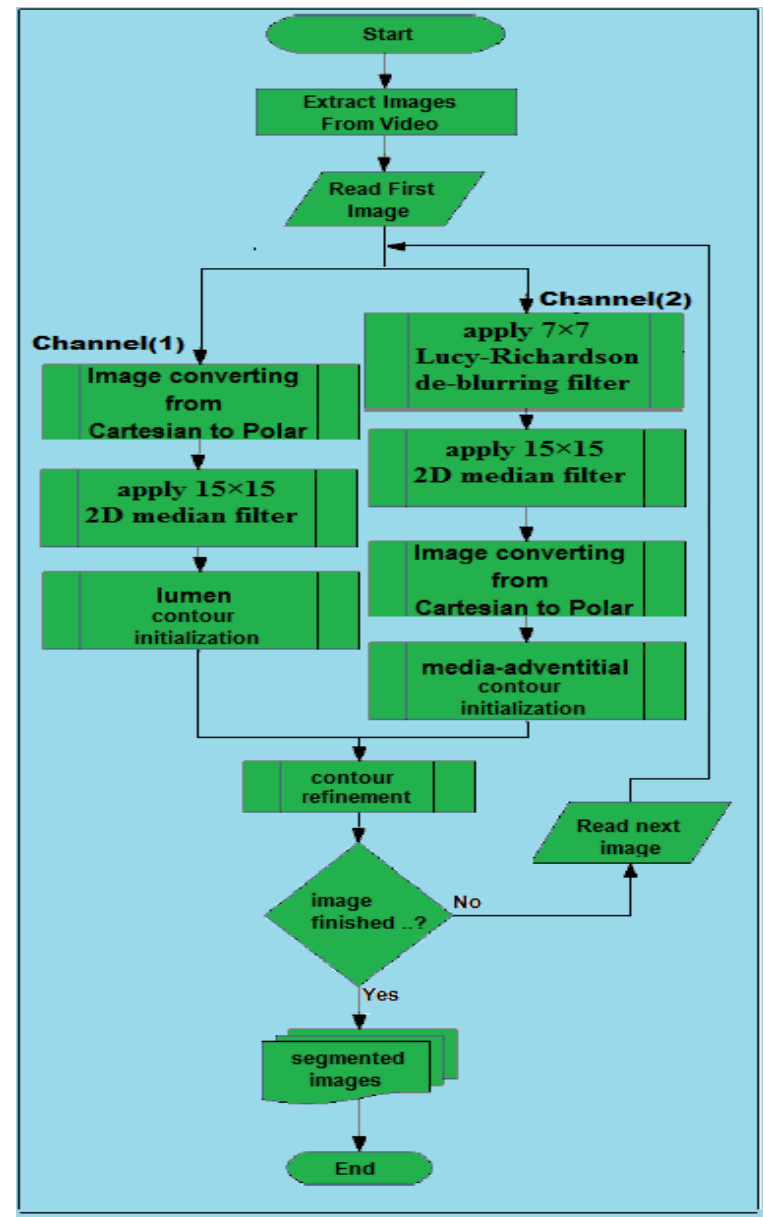

Fig. 5. The flowchart of the method (3).

\section{2) Automatic table stage}

This stage is designed to calculate LCSA, VCSA, and WCSA using the proposed algorithm. Steps of this stage are similar to manual table stage except in (b), the red contour illustrates the lumen border and blue contour illustrates the media-adventitial border.

\section{3) Statistical analysis stage}

The statistical analysis stage is used to evaluate the performance of the proposed algorithm on 92 IVUS images. These steps are as follows:

1) Convert area unit from pixel to $\mathrm{mm} 2$

2) Calculate the average area of the automatic and the manual LCSA, VCSA, and WCSA.

3) Calculate the difference in area between the automatic and the manual LCSA, VCSA, and WCSA.

4) Using steps (a) and (b) to plot the Bland-Altman diagram of the differences in area between the automated and the 
manual segmentation LCSA, VCSA, and WCSA

5) Using linear interpolation fit algorithm to plot the linear regression of the differences in area between the automated and the manual segmentation.

6) Calculate the performance factors (mean of absolute error, the standard deviation of the error, the max., and min. error).

Using previous steps to plot the error figures of LCSA, VCSA, and WCSA.

\section{RESULT AND DISCUSSION}

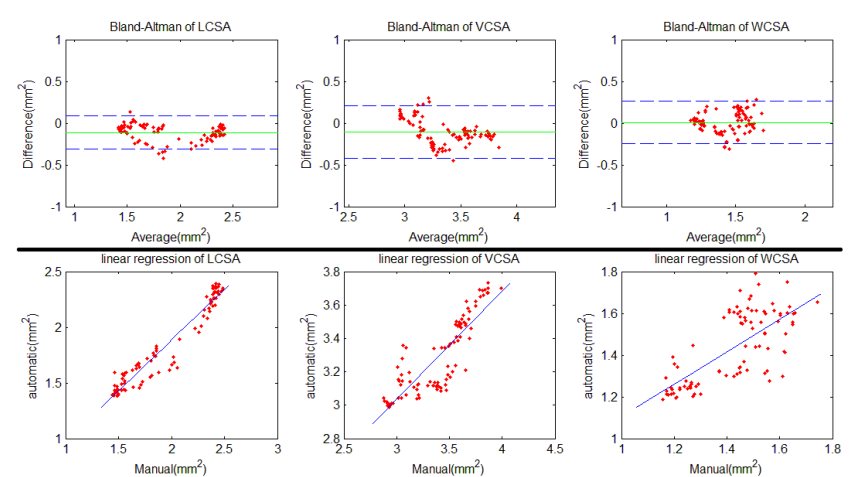

Fig. 6. The bland-altman and linear regression plots of the differences between automated (method 1) and manual segmentation, LCSA: lumen cross-sectional area, VSCA: vessel cross-sectional area, WCSA: wall cross-sectional area.

If the manual method represents the reference data which are compared with three different proposed automated methods to get the statistical analys. Table I shows the parameters of the statistical analysis. The Bland- Altman and linear regression plots are shown in Fig. 6-Fig. 8 for Method (1) to (3), for typical images acquired by the mechanical IVUS imaging system.

Table II shows error parameters. From Table II, it is found that:

Method (1) is the best method for the VCSA performance factors,
Method (2) is the best method for the LCSA performance factors, and

Method (3) is the best method for the WCSA performance factors with min error. Finally, Fig. 9 shows the standard deviation and the mean error of our methods. Fig. 10 shows the automatic segmented images, and the manually segmented images.

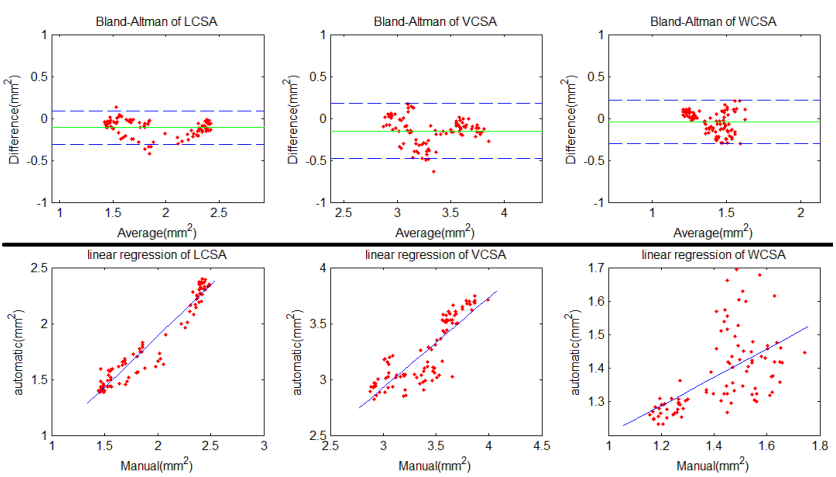

Fig. 7. The bland-altman and linear regression plots for the differences between automated (method 2) and manual segmentation, LCSA: lumen cross-sectional area, VSCA: vessel cross-sectional area, WCSA: wall cross-sectional area.

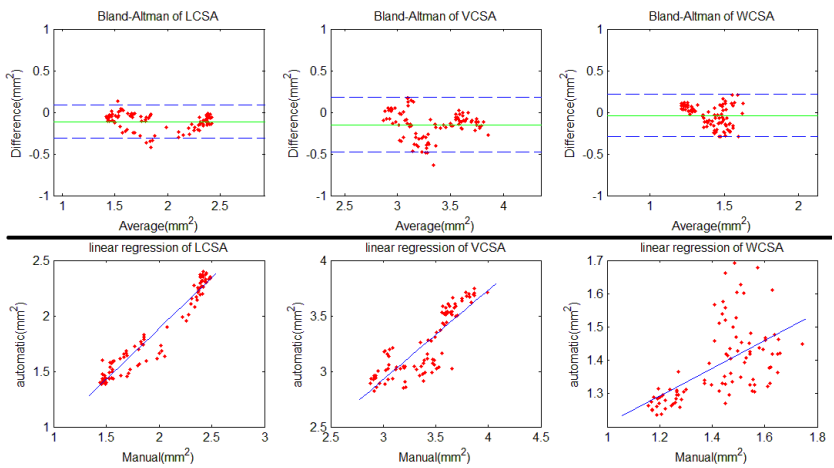

Fig. 8. The bland-altman and linear regression plots for the differences between automated (method 3) and manual segmentation, LCSA: lumen cross-sectional area, VSCA: vessel cross-sectional area, WCSA: wall cross-sectional area.

TABLE I: The ERror ANalysis to Evaluate the Three Proposed Methods

\begin{tabular}{|c|c|c|c|c|c|c|c|c|c|}
\hline & \multicolumn{3}{|c|}{ Method (1) } & \multicolumn{3}{|c|}{ Method (2) } & \multicolumn{3}{|c|}{ Method (3) } \\
\hline & LCSA & VCSA & WCSA & LCSA & VCSA & WCSA & LCSA & VCSA & WCSA \\
\hline Mean differences $\left(\mathrm{mm}^{2}\right)$ & -0.1131 & -0.1042 & 0.0089 & -0.1109 & -0.1512 & -0.0403 & -0.1125 & -0.1506 & -0.0381 \\
\hline Standard deviation & \pm 0.1004 & \pm 0.1571 & \pm 0.1257 & \pm 0.1000 & \pm 0.1640 & \pm 0.1287 & \pm 0.1001 & \pm 0.1638 & \pm 0.1285 \\
\hline Slope & 0.9140 & 0.6481 & 0.7781 & 0.9162 & 0.8072 & 0.4226 & 0.9151 & 0.8096 & 0.4180 \\
\hline intercept & 0.0567 & 1.0913 & 0.3248 & 0.0545 & 0.5037 & 0.7816 & 0.0551 & 0.4961 & 0.7904 \\
\hline
\end{tabular}

TABLE II: The ERror Analysis to Evaluate the Three PRoposed Methods

\begin{tabular}{|c|c|c|c|c|c|c|c|c|c|}
\hline & \multicolumn{3}{|c|}{ Method (1) } & \multicolumn{3}{|c|}{ Method (2) } & \multicolumn{3}{|c|}{ Method (3) } \\
\hline & LCSA & VCSA & WCSA & LCSA & VCSA & WCSA & LCSA & VCSA & WCSA \\
\hline Mean Absolute Error & 0.1189 & 0.1629 & 0.0985 & 0.1167 & 0.1752 & 0.1090 & 0.1182 & 0.1745 & 0.1090 \\
\hline Standard deviation & 0.0934 & 0.0939 & 0.0780 & 0.0930 & 0.1378 & 0.0787 & 0.0931 & 0.1378 & 0.0772 \\
\hline Max Error & 0.4217 & 0.4457 & 0.3124 & 0.4188 & 0.6327 & 0.2963 & 0.4224 & 0.6302 & 0.2953 \\
\hline Min Error & 0.0047 & 0.0026 & 0.0020 & 0.0035 & 0.0024 & 0.0006 & 0.0021 & 0.0010 & 0.0005 \\
\hline
\end{tabular}



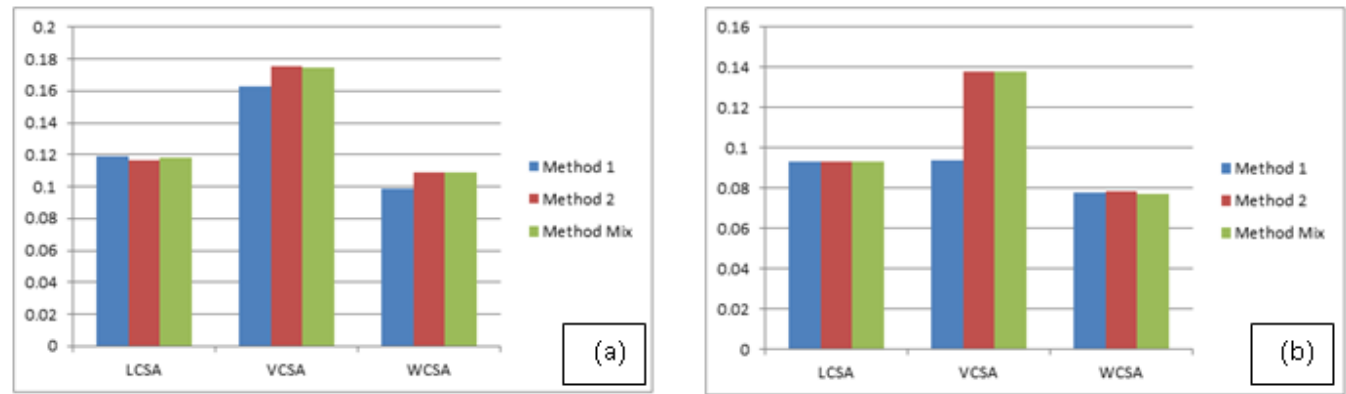

Fig. 9. The mean error a) and standard deviation b) of the three proposed methods.
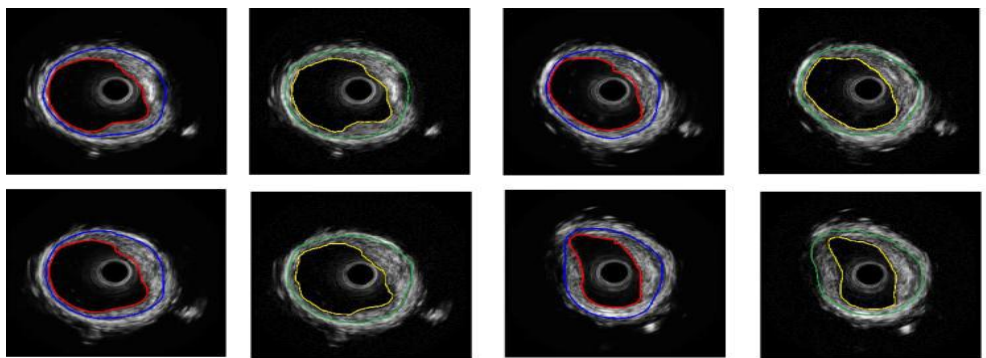

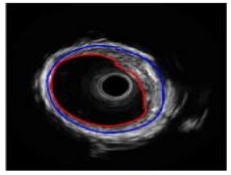

(a)

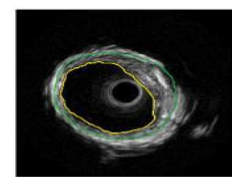

(b)

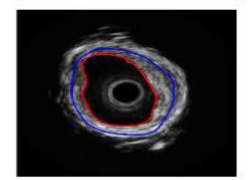

(a)

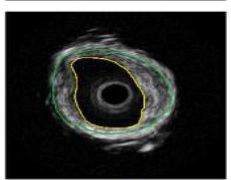

(b)

Fig. 10. The experimental results from typical images acquired with mechanical IVUS imaging system, (a) Automatic segmented image, (b) Manually segmented image.

\section{CONCLUSION}

The proposed technique is composed of three different methods for detection of lumen and media-adventitia borders in IVUS images. It is a fully automated technique. The catheter region was automatically discarded. The technique had the ability to work under artifacts and noisy conditions. The de noising of image depends on the type and position of the filter which was used. Our proposed methods experimentally evaluated in large data sets (92 frame) of IVUS images derived from human coronary arteries "iCrossTM Boston Scientific system". The fully automated test of the algorithm was designed to test our proposals performance using the MATLAB® program.

To get performance factors of VCSA, method (1) was recommended. Method (2) was recommended for LCSA, and method (3) for WCSA and it had min error. Moreover, the technique had several clinical applications. It could facilitate plaque morphometric analysis i.e. plan-metric, and wall thickness calculations.

\section{REFERENCES}

[1] World Health Rankings. (July 2013). [Online]. Available: http://www.worldlifeexpectancy.com/country-health-profile/egypt

[2] M. Schmauder, C. M. Gross, M. Firle, J. Waigand, and R. Orglmeister, "Automated detection of endovascular contours from intravascular ultrasound image sequence using a simulated annealing approach," in Proc. 8th Mediterranean Conf. on Medical and Biological Engineering and Computing, Lemesos, Cyprus, 1998.

[3] M. Papadogiorgaki, V. Mezaris, Y. Chatzizisis, G. Giannoglou, and I. Kompatsiaris, "Image analysis techniques for automated IVUS contour detection," Ultrasound in Medicine and Biology, vol. 34, pp. 1482-1498, 2008.

[4] R. Sanz-Requena, D. Moratal, D. Garca-Snchez, V. Bod, J. Rieta, and J. Sanchis, "Automatic segmentation and 3D reconstruction of intravascular ultrasound images for a fast preliminary evaluation of vessel pathologies," Computerized Medical Imaging and Graphics, vol. 31, pp. 71-80, 2007.

[5] S. E. Nissen, An atlas and manual of coronary intravascular ultrasound imaging, 1st ed., Parthenon Publishing Group, U.K.: London, 2004.

[6] W. Li, C. V. Birgelen, C. D. Mario, E. Boersma, E. J. Gussenhoven, N V. D. Putten, and N. Bom, "Semi-automatic contour detection for volumetric quantification of intracoronary ultrasound," in Proc. IEEE on Computers in Cardiology, Bethesda, Maryland, pp. 277-280, 1994.

[7] M. Sonka, X. Zhang, M. Siebes, M. S. Bissing, S. C. Dejong, S. M. Collins, and C. R. McKay, "Segmentation of intravascular ultrasound images: a knowledge-based approach," IEEE Trans. on Medical Imaging, vol. 14, pp. 719-732, 1995.

[8] C. V. Birgelen, C. Mario, W. Li, J. Schuurbiers, C. Slager, P. D. Feyter, J. Roelandt, and P. Serruys, "Morphometric analysis in three dimensional intracoronary ultrasound: an in vitro and in vivo study using a novel system for the contour detection of lumen and plaque," American Heart Journal, vol. 132 no. 3, pp. 516-527, 1996.

[9] R. Shekhar, R. M. Cothren, D. G. Vince, S. Chandra, J. D. Thomas, and J. F. Cornhill, "Three-dimensional segmentation of luminal and adventitial borders in serial intravascular ultrasound images," Computerized Medical Imaging and Graphics, vol. 23 no. 6, pp. 299-309, 1999.

[10] J. Dijkstra, G. Koning, J. Tuinenburg, P. Oemrawsingh, and J. Reiber, "Automatic border detection in intravascular ultrasound images for quantitative measurements of the vessel, lumen and stent parameters," Computer Assisted Radiology and Surgery, vol. 1230, pp. 916-922, 2001.

[11] M. E. Plissiti, D. I. Fotiadis, L. K. Michalis, and G. E. Bozios, “An automated method for lumen and media-adventitia border detection in a sequence of IVUS frames," IEEE Transactions on Information Technology in Biomedicine, vol. 8, pp. 131-141, 2004.

[12] D. M. Herrington, T. Johnson, P. Santago, and W. E Snyder, "Semi-automated boundary detection for intravascular ultrasound," in Proc. IEEE on Computers in Cardiology, 1992, vol. 192, pp. 103-106.

[13] E. G. Mendizabal-Ruiz, M. Rivera, and I. A. Kakadiaris, "A probabilistic segmentation method for the identification of luminal borders in intravascular ultrasound images," in Proc. IEEE Computer Society Conference on Computer Vision and Pattern Recognition, Anchorage, AK, pp. 1-8, 2008.

[14] M. Cardinal, G. Soulez, J. C. Tardif, J. Meunier, and G. Cloutier, "Fast-marching segmentation of three-dimensional intravascular ultrasound images: a pre-and post-intervention study," Medical Physics, vol. 37, no. 7, pp. 3633-3647, 2010. 
[15] Z. Luo, Y. Wang, and W. Wang, "Estimating coronary artery lumen area with optimization-based contour detection," IEEE Trans. on Medical Imaging, vol. 22, no.4, pp. 564-566, 2003.

[16] E. Brusseau, C. L. D. Korte, F. Mastik, J. Schaar, and A. F. W. V. der Steen, "Fully automatic luminal contour segmentation in intracoronary ultrasound imaging - a statistical approach," IEEE Trans. on Medical Imaging, vol. 23, no. 5, pp. 554-566, 2004.

[17] D. Gil, A. Hernandez, O. Rodriguez, J. Mauri, and P. Radeva, "Statistical strategy for anisotropic adventitia modelling in IVUS," IEEE Trans. on Medical Imaging, vol. 25, pp. 768-778, 2006.

[18] M. Zheng, W. Yubin, W. Yousheng, S. Xiaodi, and W. Yali, "Detection of the lumen and media-adventitia borders in IVUS imaging," in Proc. 9th International Conf. on Signal Processing, vol. 2 pp. 1059-1062, 2008.

[19] A. Katouzian, E. D. Angelini, S. G. Carlier, J. S. Suri, N. Navab, and A. F. Laine, "A state-of-the-art review on segmentation algorithms in intravascular ultrasound (IVUS) images," IEEE Transactions on Information Technology in Biomedicine, vol. 16, no. 5, pp. 823-834, 2012.

[20] M. M. Amine and H. M. Ali, "Intravascular Ultrasound Image Segmentation Using Morphological Snakes," International Journal of Image, Graphics and Signal Processing (IJIGSP), vol. 4, no. 5, pp. 54-60, 2012.

[21] E. G. Mendizabal-Ruiz, M. Rivera, and I. A. Kakadiaris, "Segmentation of the luminal border in intravascular ultrasound B-mode images using a probabilistic approach," Medicallmage Analysis, vol. 17, pp. 649-670, 2013.

[22] M. C. Moraes and S. S. Furuie, "An automatic media-adventitia border segmentation approach for IVUS images," in Proc. IEEE on Computing in Cardiology, pp. 389-392, 2010.

[23] F. Ciompi, O. Pujol, C. Gatta, M. Alberti, S. Balocco, X. Carrillo, J. Mauri, and P. Radeva, "HoliMAb: A holistic approach fo Media-Adventitia border detection in intravascular ultrasound," Medical Image Analysis, vol.16, pp. 1085-1100, 2012.

[24] M. Olszewski, A. Wahle, S. Mitchell, and M. Sonka, "Segmentation of intravascular ultrasound images: a machine learning approach mimicking human vision," International Congress Series, vol. 1268 , pp. 1045-1049, 2004

[25] C. J. Bouma, W. J. Niessen, K. J. Zuiderveld, E. J. Gussenhoven, and M. A. Viergever, "Automated lumen definition from $30 \mathrm{MHz}$ intravascular ultrasound images," Medical Image Analysis, vol. 1, no. 4, pp. 363-377, 2012.

[26] G. Unal, S. Bucher, S. Carlier, G. Slabaugh, T. Fang, and K. Tanaka, "Shape-driven segmentation of the arterial wall in intravascula ultrasound images," IEEE transactions on information technology in biomedicine, vol. 12, pp. 335-347, 2008.

[27] M. Alberti, S. Balocco, C. Gatta, F. Ciompi, O. Pujol, J. Silva, X. Carrillo, and P. Radeva, "Automatic bifurcation detection in coronary IVUS sequences," IEEE Trans. on Medical Engineering, vol. 59, no. 4 pp. 1022-2031, 2012.

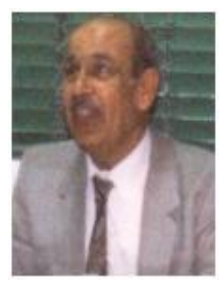

Mazhar B. Tayel was born in Alexandria, Egypt on Nov. 20th, 1939. He was graduated from Alexandria University, Faculty of Engineering Electrical and Electronics Department Class in 1963. He published many papers and books in electronics, biomedical, and measurements.

Prof. Dr. Mazhar Bassiouni Tayel had his B.Sc. with honor degree in 1963, and then he had his Ph.D. electro-physics degree in 1970 . He had this Prof degree of elect. and communication and biomedical engineering and systems in 1980. Now he is Emirate. Prof. since 1999.

From 1987 to 1991 he worked as a chairman, communication engineering section, EED BAU-Lebanon and from 1991 to 1995 he worked as the chairman of Communication Engineering Section, EED Alexandria University, Alexandria Egypt, and from 1995 to 1996 he worked as the chairman of EED, Faculty of Engineering, BAU-Lebanon, and from 1996 to 1997 he worked as the dean of the Faculty of Engineering, BAU - Lebanon, and from 1999 to 2009 he worked as a senior professor in the Faculty of Engineering, Alexandria. University, Alexandria Egypt, finally from 2009 to now he worked as emirate professor in the Faculty of Engineering, Alexandria University, Alexandria Egypt. Prof. Dr. Tayel worked as a general consultant in many companies and factories also he is Member in supreme consul of Egypt.

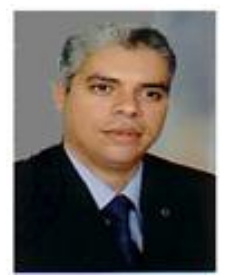

M. A. Massoud was born in Alexandria, Egypt on September 8th, 1964. He was graduated from Alexandria University Faculty of Engineering Electrical and Electronics department class 1987. He published many papers in biomedical application.

Dr. Mohamed Ahmed Massoud's major field is biomedical engineering and instrumentation, he had his B.Sc. in 1987, and then he had his master degree in 1999 and then he had his Ph.D. degree in 2004.

From 1987 to 2004 he worked as a control engineer Alexandria-Egypt, and from 2004 to now he worked as a lecture in El-Minia University, Faculty of Engineering, BED, El-Minia, Egypt.

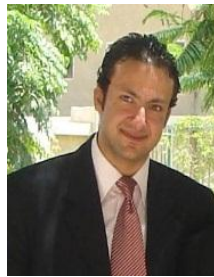

Y. F. Shehata was born in Alexandria, Egypt on August 5th, 1987. He was graduated from El-Minia University, Faculty of Engineering Computers and Systems Department, class 2009. He is a post graduate student.

Eng. Yasseen Farouk Shehata's major field is software engineering and systems; he had his B.Sc. in 2009.

From 2009 to now he worked as researcher in E -Minia University, Faculty of Engineering, CSED, El-Minia, Egypt. 